\title{
Identification and abiotic stress response of a glutamine synthetase gene (AccGS) from the Asiatic honeybee, Apis cerana cerana (Hymenoptera: Apidae)
}

\author{
XIULING WANG ${ }^{1}$, YuZHen $\mathrm{LI}^{1}$, YAN YAN ${ }^{1}$, BAOHUA XU ${ }^{2 *}$ and XINGQI GUO ${ }^{1 *}$ \\ ${ }^{1}$ College of Life Sciences and ${ }^{2}$ College of Animal Science and Technology, Shandong Agricultural University, Taian, \\ Shandong, 271018, P.R. China; e-mails: seabiscuit_616@126.com (X.Wang), liyuzhen1988@163.com (Y. Li), \\ sdytyanyan@163.com (Y. Yan),xqguo@sdau.edu.cn (X. Guo), bhxu@sdau.edu.cn (B. Xu)
}

Key words. Hymenoptera, Apidae, Apis cerana cerana, glutamine synthetase, expression analysis, stress response

\begin{abstract}
Glutamine synthetase (GS) is an essential detoxification enzyme that plays an important role in stress responses; however, little information regarding the function of this enzyme in hymenopteran insects is available. In the present study, we isolated and characterized the gene encoding GS in the Asiatic honeybee, Apis cerana cerana. Multiple alignments and a phylogenetic analysis of GS sequences showed that AccGS belongs to the GSII superfamily and clusters with invertebrate GSs. Real-time quantitative PCR data demonstrated that $A c c G S$ is expressed at all developmental stages and in all tissues, with the highest expression observed in the sixth larval instar and in the brain. Moreover, $A c c G S$ expression is highly regulated by environmental stress, including xenobiotic, temperature, and ultraviolet light stresses. A disc diffusion assay showed that the recombinant AccGS protein confers resistance to mercuric chloride $\left(\mathrm{HgCl}_{2}\right)$ stress in E. coli. This suggests that AccGS may play multiple roles in early development and in environmental stress responses.
\end{abstract}

\section{INTRODUCTION}

Glutamine synthetase (GS), also known as the molecular clock, has been described as the oldest extant, functional gene in the history of evolution (Kumada et al., 1993). GS participates in many biological processes, such as cell growth, energy metabolism, protein and nucleotide synthesis, and immune responses (Lai et al., 2011). Although all cells express GS to some extent, the expression of this gene is controlled in a developmental stage- and tissue-specific manner (He et al., 2010). GSs are classified into three distinct forms: GSI, which is present only in prokaryotes; GSII, found in eukaryotes and some prokaryotes; and GSIII, which is present in anaerobes (Kinoshita et al., 2009).

As a member of a phylogenetically well-conserved subfamily of GSs, GSII contains five conserved regions, $\mathrm{NH}_{4}{ }^{+}$-binding residues, ATP-binding residues, and glutamate-binding residues (Qiu et al., 2012). GSII is a key enzyme in nitrogen metabolism and plays a major role in ammonia detoxification, acid-base homeostasis, and cell signaling (Häberle et al., 2005). In vertebrates, one of the main roles of GSII is to metabolize glutamate and ammonia to glutamine $\left(\mathrm{Glu}+\mathrm{NH}_{3}+\mathrm{ATP} \leftrightarrow \mathrm{Gln}+\right.$ $\mathrm{ADP}+\mathrm{Pi})$. This reaction is biologically significant because GS is the only enzyme capable of the de novo glutamine biosynthesis of glutamine, a compound that is important in several cell-specific processes (He et al., 2010).

Studies on GS enzymes have focused mainly on their roles in detoxification and environmental stress responses. Most of the physiological responses to stressors ulti- mately generate ammonia, which is partly responsible for activating GS (Tanguy et al., 2005b). Providing exogenous active GS can help to enhance ammonia detoxification and reduce neurological damage (Kosenko et al., 2008). Pesticide metabolites and degradation products can be as toxic as their respective parent compounds and are often more systemic (Mullin et al., 2010). When metabolized, some pesticides release nitrogen as an ammonium group, which can activate GS (Geret et al., 2013), and one strategy for increasing ammonia detoxification is to increase its incorporation into glutamine by GS (Kosenko et al., 2008). Additionally, glutamine functions in a variety of biochemical functional processes. Glutamine is conditionally essential and has been shown to be a key pharmaconutrient in stress and injury responses (Weitzel $\&$ Wischmeyer, 2010). Glutamine can function as a metabolic fuel and stress-signaling molecule in illness and injury via the heat shock protein (HSP), the expression of which is vital to cellular and tissue protection during stress and injury (Liu \& Chen, 2011). Moreover, in glutamine-poor media, GS activity is necessary for HSP expression in fruit fly, Drosophila, Kc cells (Sanders \& Kon, 1992). Because glutamine is synthesized by GS, it is essential for stress responses.

The Asiatic honeybee, Apis cerana cerana Fabricius plays an important role in maintaining the biodiversity of plants and can effectively pollinate mountain plants as well as crops (Xu et al., 2009). Environmental toxicity is a key factor that influences biological pathways in organisms, and the protection of honeybee species is a concern worldwide. In the present study, we identified the GS gene in $A$. cerana cerana (AccGS), analyzed its develop-

\footnotetext{
* Corresponding authors.
} 
TABLE 1. The primers used in this experiment.

\begin{tabular}{lll}
\hline Primer name & Description & Primer sequence (5'-3') \\
\hline QP1 & cDNA sequence primer, forward & CAACATAACACGATAAGACGCG \\
QP2 & cDNA sequence primer, reverse & GGTACGATCACTATTGCACTAC \\
5P1 & 5' RACE reverse primer, primary & GTTGAGGCAGGTCGAGGTATTAT \\
AAP & 5' RACE reverse primer, nested & GGTTTTGTCTAAGGCCGCATA \\
AUAP & Abridged Anchor Primer & GGCCACGCGTCGACTAGTAC(G) ${ }_{16}$ \\
3P1 & Abridged universal amplification primer & GGCCACGCGTCGACTAGTAC \\
3P2 & 3' RACE reverse primer, primary & TGCACATACAAATTTCTCGAC \\
B25 & 3' RACE reverse primer, nested & ACGTCTGACTGGAAAATGCG \\
B26 & Universal primer, nested & GACTCTAGACGACATCGA \\
GU1 & Universal primer, primary & GACTCTAGACGACATCGA(T) ${ }_{17}$ \\
GD2 & 5' flanking-region primer, forward & TGTTTGTTCGATAATTGTTCAGT \\
GYH1 & 5' flanking-region primer, reverse & ACTCTACACGATTGCTGGA \\
GYH2 & Protein expression primer, forward & GGATCCATGTCACGTGTCATACTGAAG \\
QRT1 & Protein expression primer, reverse & CTCGAGTTCATTTAGGACACAAGTGCG \\
QRT2 & Q-PCR primer, forward & CACACTCTACACGATTGCTGGAC \\
$\beta$-s & Q-PCR primer, reverse & TTTTCCGGTTGAGGCAGGT \\
$\beta-x$ & Standard control primer, forward & TTATATGCCAACACTGTCCTTT \\
\hline
\end{tabular}

ment and tissue expression pattern, and examined its expression in response to environmental stress.

\section{MATERIAL AND METHODS}

\section{Insect material and treatments}

Asiatic honeybees, A. cerana cerana used in this study were bred at the experimental apiary of Shandong Agricultural University, Tai'an, China. Various tissues from two-week-old adult worker honeybees, including the head, thorax, abdomen, muscle, epidermis, and midgut, were freshly dissected and collected. To detect AccGS expression patterns under environmental stress, two-week-old adult worker honeybees were collected from combs in outdoor beehives, divided into groups, and separately treated with cold $\left(4^{\circ} \mathrm{C}\right)$, heat $\left(42^{\circ} \mathrm{C}\right)$, ultraviolet (UV) light $\left(30 \mathrm{~mJ} / \mathrm{cm}^{2}\right)$, or hydrogen peroxide $\left(\mathrm{H}_{2} \mathrm{O}_{2}\right)$ (30\%; Kaitong, Tianjin, China). For the pesticide-herbicide treatments, the following compounds: cyhalothrin, paraquat, acetamiprid, acaricides, and phoxim were diluted to $12.5 \mathrm{mg} / \mathrm{l}$, $20 \mathrm{mg} / 1,20 \mathrm{mg} / 1,20 \mathrm{mg} / 1$, and $20 \mathrm{mg} / 1$, respectively. To analyze the influence of heavy metals on AccGS expression, a pollen and sucrose solution containing mercuric chloride $\left(\mathrm{HgCl}_{2}\right)(1.5$ $\mu \mathrm{g} / \mathrm{ml}$ ) was fed to the adult workers. The treated honeybees were immediately frozen in liquid nitrogen at the indicated time points and stored deep frozen at $-80^{\circ} \mathrm{C}$ until RNA extraction.

\section{RNA extraction, cDNA synthesis, and DNA isolation}

Total RNA was extracted from the treated bees using Trizol reagent (Invitrogen, Carlsbad, CA, USA) according to the manufacturer's protocol. EasyScript First-Strand cDNA Synthesis SuperMix (TransGen Biotech, Beijing, China) was used for cDNA synthesis according to the manufacturer's instructions. Genomic DNA was isolated from the adult bees using an EasyPure Genomic DNA Extraction Kit (TransGen Biotech, Beijing, China).

\section{Isolation of the cDNA and 5'-flanking region of AccGS}

To determine the cDNA sequence of $A c c G S$, cloning was performed as described previously (Meng et al., 2011). The 5'- flanking region of $A c c G S$ was amplified according to the genomic sequence of $A$. mellifera. The primer sequences used are shown in Table 1. The putative cis-acting elements and transcription factor-binding sites in the 5'-flanking region of AccGS were predicted using the Matlnspector database (http://www.cbrc.jp/research/db/TFSEARCH.html).

\section{Bioinformatic analysis and phylogenetic tree construction}

A multiple alignment analysis of GS was conducted with DNAman software, version 5.2.2 (Lynnon Biosoft Company, Quebec, Canada). The conserved domains in AccGS were identified using the bioinformatic tools available on the NCBI server (http://blast.ncbi.nlm.nih.gov/Blast.cgi). The theoretical isoelectric point and molecular weight of AccGS were predicted by PeptideMass (http://web.expasy.org/peptide mass/). The phylogenetic tree was constructed using the neighbour-joining method in MEGA version 4.1 (http://megasoftware.net).

\section{Recombinant AccGS protein overexpression and disc diffu- sion assay}

The AccGS coding region was subcloned from the pEasy-T1 vector into the pET-30a $(+)$ expression vector after digestion with the restriction endonucleases $B a m \mathrm{HI}$ and $\mathrm{XhoI}$. The recombinant plasmid pET-30a (+)-AccGS was transformed into $E$. coli BL21 (DE3) cells, which were grown at $37^{\circ} \mathrm{C}$ in Luria-Bertani (LB) medium containing $50 \mathrm{mg} / \mathrm{ml}$ kanamycin. After the cell density reached 0.8 at optical density $(\mathrm{OD})_{600}$, isopropyl 1-thio- $\beta$-galactopyranoside (IPTG) was added at a final concentration of $0.4 \mathrm{mM}$ to induce the production of recombinant protein and induction for $8 \mathrm{~h}$. Approximately $5 \times 10^{8}$ cells from each culture were plated on LB-kanamycin agar plates and then incubated at $37^{\circ} \mathrm{C}$ for $1 \mathrm{~h}$. Filter discs (6-mm diameter) soaked with different concentrations of $\mathrm{HgCl}_{2}(0,0.2,0.4,0.6,0.8,1,1.5 \mathrm{~g} / \mathrm{l})$ were placed on the surface of the agar. The cells were grown for $24 \mathrm{~h}$ at $37^{\circ} \mathrm{C}$, and the growth inhibition zones around the paper discs were measured. 

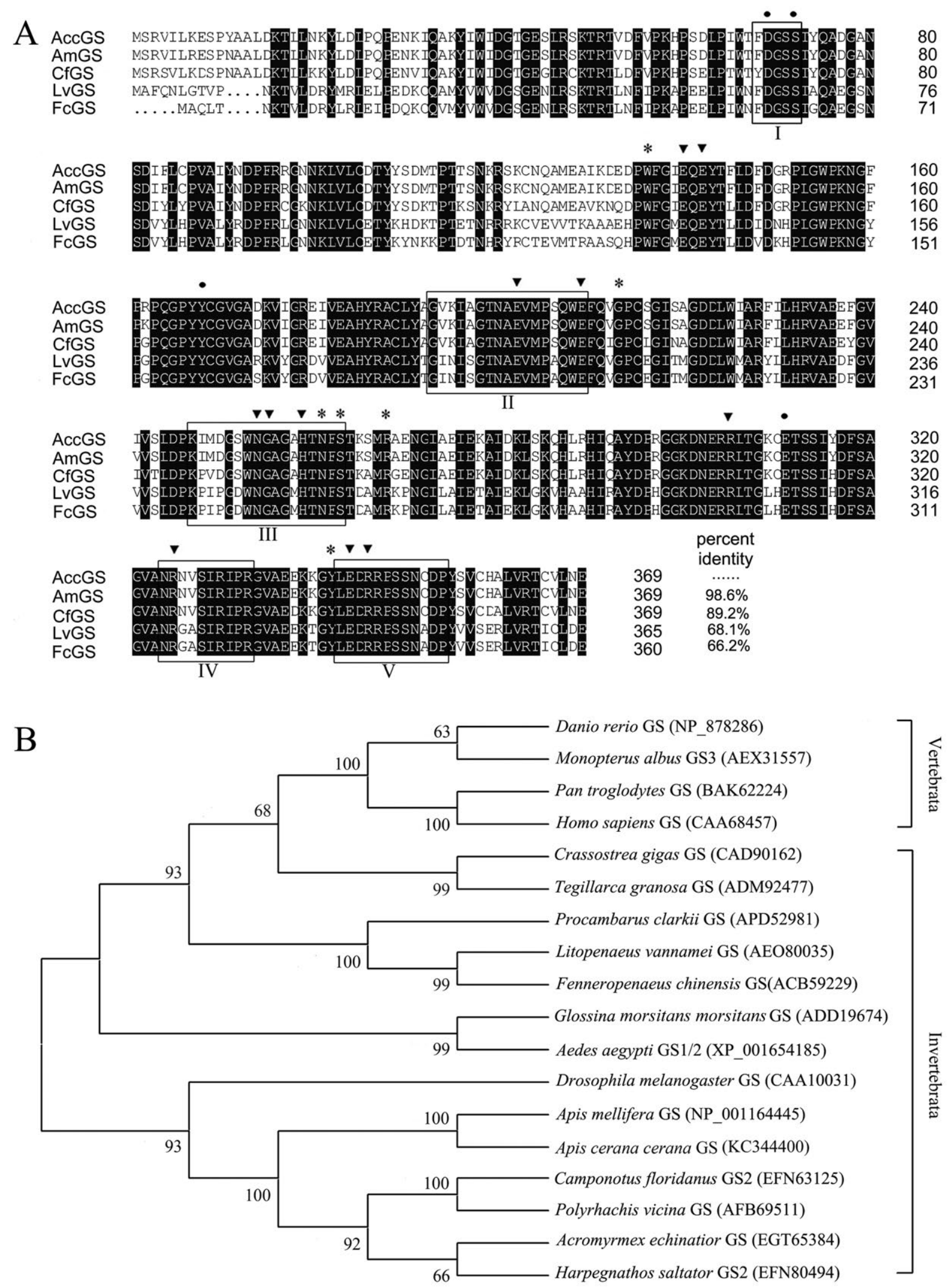

Fig. 1. AccGS sequence analysis. A - comparison of the deduced amino acid sequences of GSs in different animal species. Abbreviations: Acc - Apis cerana cerana; Am - Apis mellifera; Cf - Camponotus floridanus; Lv - Litopenaeus vannamei; Fc - Fenneropenaeus chinensis. The five conserved subdomains are boxed and labelled with Roman numerals (I-V). The $\mathrm{NH}_{4}{ }^{+}$-binding residues, ATP-binding residues, and glutamate-binding residues of GS are marked with dots $(\bullet)$, asterisks $(*)$, and arrowheads $(\mathbf{v})$, respectively. B - phylogenetic tree of GSs from various species generated by the neighbour-joining method using MEGA 4.1. The numbers above or below the branches indicate the bootstrap values $(>50 \%)$ from 500 replicates. 
NIT2

TTGATAAAACATATTGCACTAAAgAAgGTAAATTATTTTGAAgATATTATTAATAATGAAAAAA

NIT2

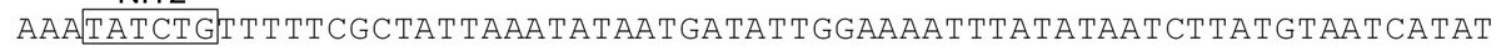

GAAAATTGAтAgATAACTTATACAAATgTAgTAATAATAgCAAAAAAATATAgAATAAATTGTTCT

HSF

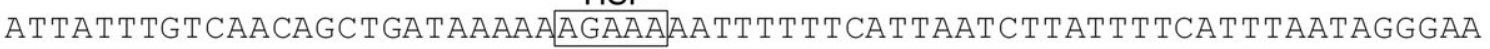

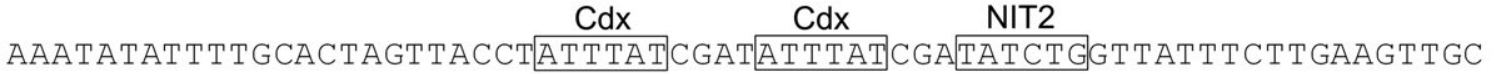

TCCGTTTGAAAATCATCTAGTAGGATTGGAAAAATTGTTGTTAAAACTTCCACACGATAATCAG

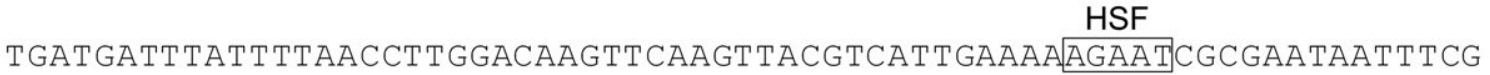

GTCGATGGTTCGTAACTGACAAGTACCATGGTAACAGAGGCAGACAAGAATTTTCCGGCATGTAACG

Fig. 2. Nucleotide sequences and putative cis-acting elements of the 5'-flanking region of AccGS. The transcription start site and translation start site are both marked with arrowhead. The transcription factor binding sites mentioned in the text are boxed.

\section{Quantitative real-time PCR (qPCR)}

The primer pair QP1 and QP2 was designed based on the cDNA sequence of $A c c G S$. The primer pair $\beta-\mathrm{s} / \beta-\mathrm{x}$ was designed based on A. mellifera $\beta$-actin (XM640276) and used as an internal control. The PCR conditions were as follows: $95^{\circ} \mathrm{C}$ for $30 \mathrm{~s}, 40$ cycles of amplification $\left(95^{\circ} \mathrm{C}\right.$ for $5 \mathrm{~s}, 55^{\circ} \mathrm{C}$ for $15 \mathrm{~s}$, and $72^{\circ} \mathrm{C}$ for $15 \mathrm{~s}$ ), and a melting curve cycle from $65^{\circ} \mathrm{C}$ to $95^{\circ} \mathrm{C}$. CFX Manager Software version 1.1 (Bio-Rad, USA) was used to analyze the data, and significant differences were evaluated using Statistical Analysis System (SAS) version 9.1 software (SAS Institute, Cary, NC). All samples were processed in three technical replicates.

\section{RESULTS}

\section{Sequence and phylogenetic analysis of $A c c G S$}

PCR amplification of the full-length cDNA sequence of GS (acc. no. KC344400) from A. cerana cerana produced a product of $1,429 \mathrm{bp}$ containing a $1,107 \mathrm{bp}$ open reading frame (ORF), a 180 bp 5' untranslated region (UTR), and a 139 bp 3' UTR. The predicted protein encoded by $A c c G S$ consisted of 369 amino acids, with a calculated molecular weight of $41.262 \mathrm{kDa}$ and an isoelectric point (pI) of 6.85. The National Center for Biotechnology Information (NCBI; http://www.ncbi.nlm.nih.gov/) website was used to analyze the basic biological characteristics of AccGS. Our search results indicated that the amino acid sequence of AccGS contains a $\beta$-grasp domain ( $E$ value, $2.57 \mathrm{e}-15$, pfam03951) and a catalytic domain $(E$ value, 2.22e-76, pfam00120). An NCBI Protein BLAST search indicated that Apis mellifera GS (AmGS; acc. no. NP_001164445) was the best match for this protein sequence, showing 98\% identity. In addition, multiple alignments showed that there are five conserved regions in the AccGS protein (Fig. 1A). These results provide additional evidence that GSs are evolutionary conserved 

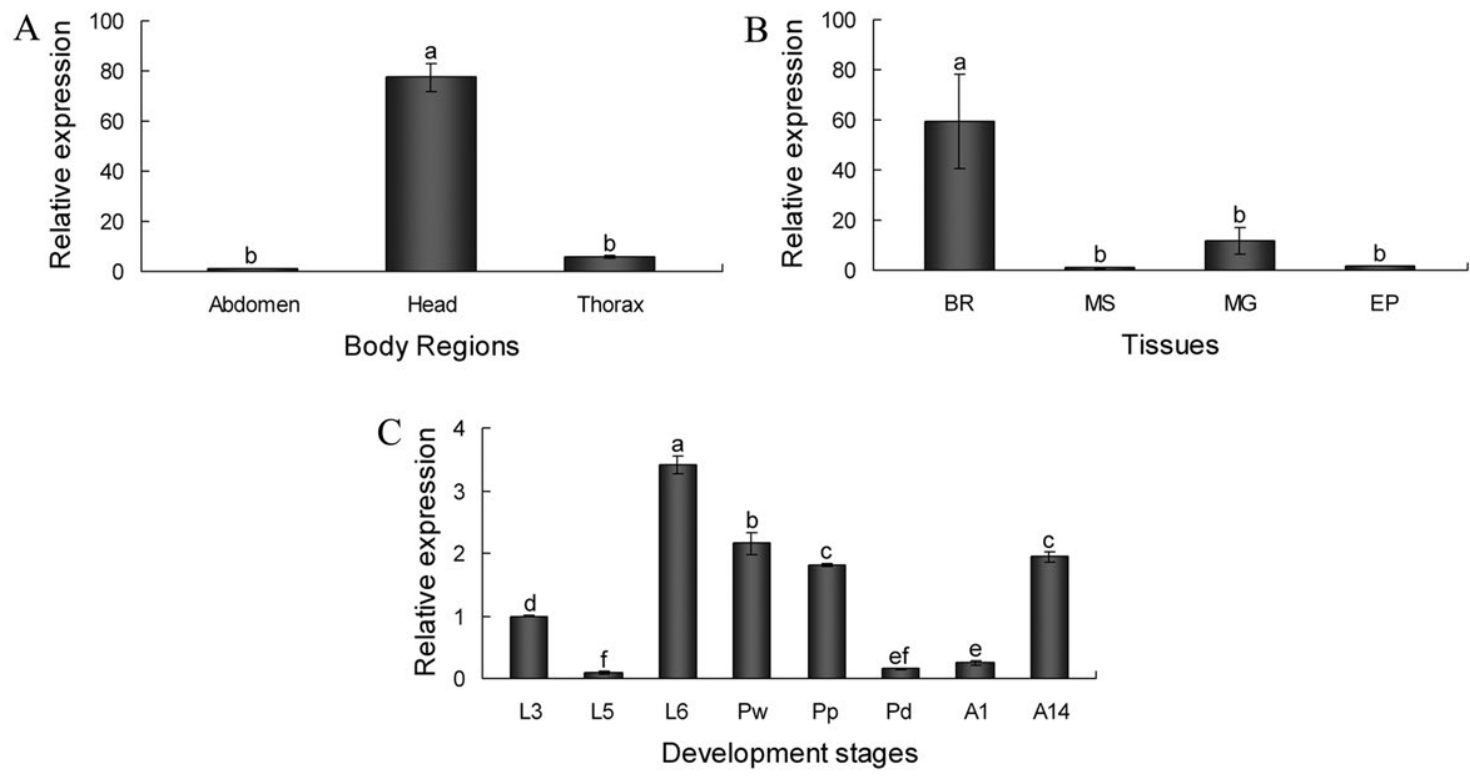

Fig. 3. AccGS expression profiles. The relative expression of $A c c G S$ was analyzed in different body regions (A) and tissues (B) and at different developmental phases (L3 - third instar larvae; L5 - fifth instar larvae; L6 - sixth instar larvae; Pw - white-eyed pupae; Pp - pink-eyed pupae; Pd - dark-eyed pupae; A1 - one-day post-emergence adults; A14 - fourteenth day post-emergence adults) $(C)$ by qPCR. The vertical bars indicate the mean \pm S.E.M. $(n=3)$. The different letters above the columns indicate significant differences $(P<0.01)$ according to Duncan's multiple range tests, calculated using SAS version 9.1 software.

among different organisms and that AccGS belongs to the GSII subfamily.

To further investigate the evolutionary relationship of AccGS with other GSs, 18 known invertebrate and vertebrate GS amino acid sequences were chosen to generate a phylogenetic tree by the neighbour-joining method using MEGA4.1. As shown in Fig. 1B, AccGS was classified as an invertebrate GS and is closely related to AmGS.

\section{Analysis of the partial putative AccGS promoter}

To examine the functions of the $A c c G S$ in the growth and development of honeybees and under environmental stresses, I-PCR was used to isolate the AccGS 5' flanking region (acc. no. KC344401). A group of TF binding sites, including Cro, HSFs, NIT2, and Cdx sites, was identified in this region using the TFSEARCH program (Fig. 2). Cro is a brain-specific transcriptional activator binding site for the CROC protein, which plays an important role in the regulation of early brain development, neuronal development, and neuronal plasticity ( $\mathrm{Fu} \&$ Marzluf, 1990). NIT2 is the binding site of the nit-2 gene in the fungus Neurospora, which encodes the major positive regulatory protein that drives the expression of nitrogen structural genes under nitrogen-limited conditions (Jeffrey et al., 2000). A HSFs (heat-induced transcriptional activators) binding motif (Fernandes et al., 1994) was also identified in the AccGS 5 ' flanking region. Moreover, the $A c c G S$ promoter contains a binding site for the caudal-related homeobox $(\mathrm{Cdx})$ transcription factor, which regulates tissue-specific expression (Ericsson et al., 2006). These data suggest that $A c c G S$ might play roles in the response to some environmental stresses and the developmental regulation of $A$. c. cerana.

\section{Expression analysis of $A c c G S$ showing tissue- and developmental stage-specific differences}

qPCR was employed to examine whether the expression of the AccGS gene was tissue specific. First, we detected AccGS expression in different body regions (Fig. 3A): relative to the expression in the abdomen, $A c c G S$ transcription was higher in the head and thorax (77.5-fold and 5.8-fold, respectively). We then examined $A c c G S$ expression in different tissues (Fig. 3B). The results indicate that the mRNA levels of $A c c G S$ in the brain, midgut, and epidermis were 59.4-, 11.5-, and 1.6-fold higher, respectively, than those in the muscle.

To investigate the developmental expression patterns of $A c c G S$, RNA extracted from every developmental stage was used to detect the stage-specific expression profile of $A c c G S$. As shown in Fig. 3C, the AccGS mRNA level decreased from the third to the fifth instar larval stages, followed by a marked increase at the sixth instar stage. $A c c G S$ expression gradually decreased in the pupal stage and then increased in 14-day-old adults. These results suggest that $A c c G S$ has important functions in specific developmental stages and tissues.

\section{Expression profiles of $A c c G S$ under environmental stress}

To determine whether $A c c G S$ is associated with different environmental stressors, worker bees were subjected to several stress conditions, and $\mathrm{qPCR}$ was employed to detect the relative mRNA levels of AccGS in response to different stressors. As shown in Fig. 4A-D, $A c c G S$ expression was sharply increased at $1 \mathrm{~h}$ following cold treatment, whereas heat treatment caused $A c c G S$ mRNA to accumulate from $1.5 \mathrm{~h}$ to $2 \mathrm{~h}$. In contrast, only 


\section{A}

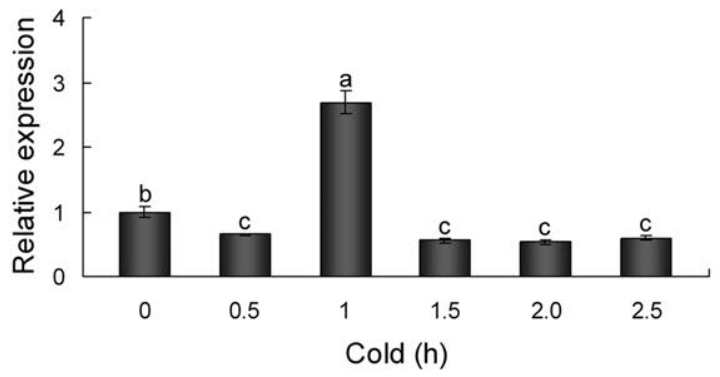

C

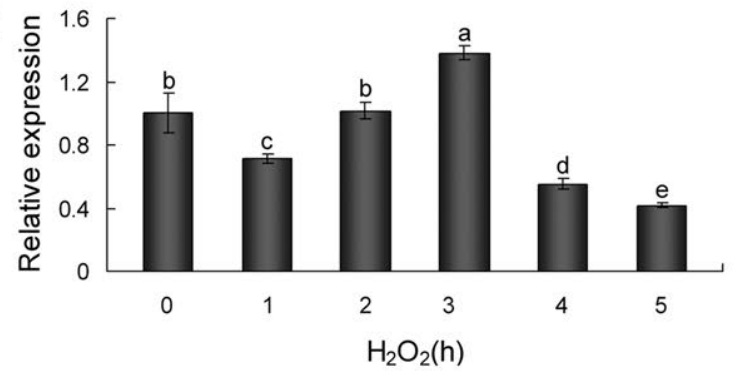

E

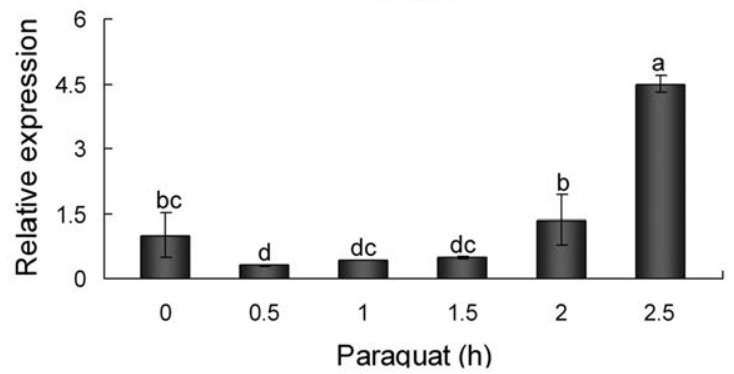

G

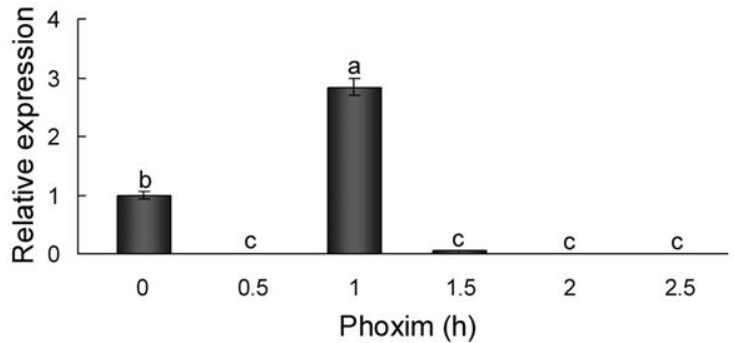

I

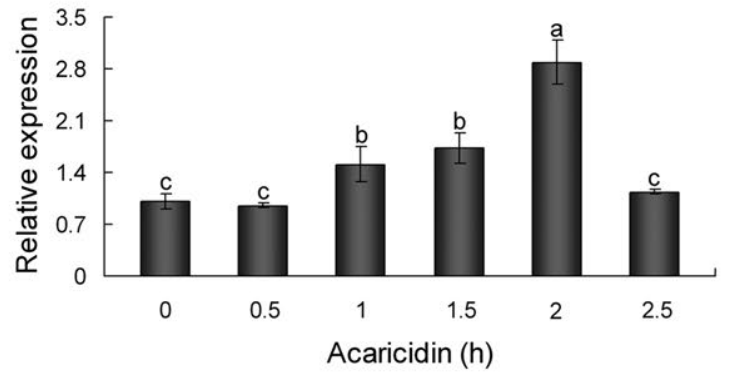

B

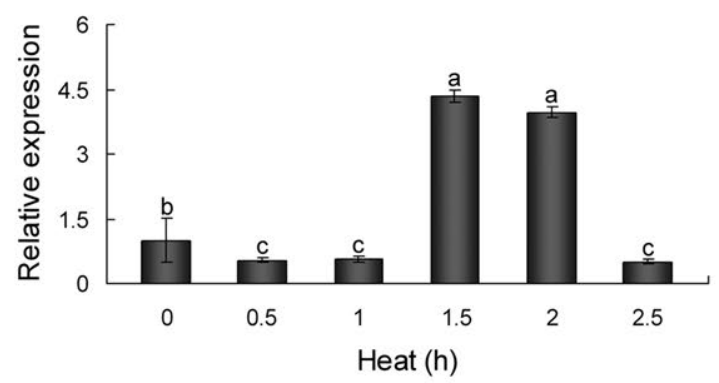

D

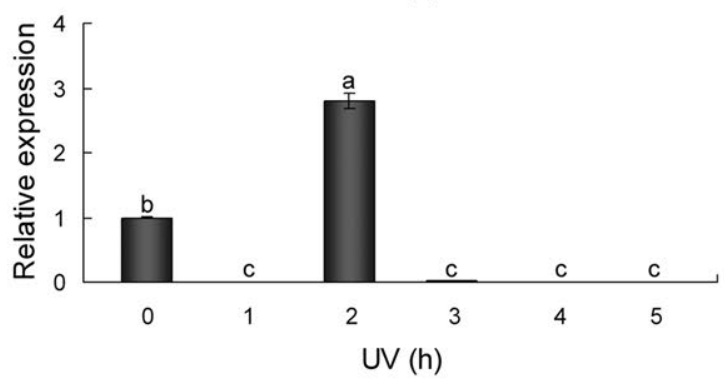

F
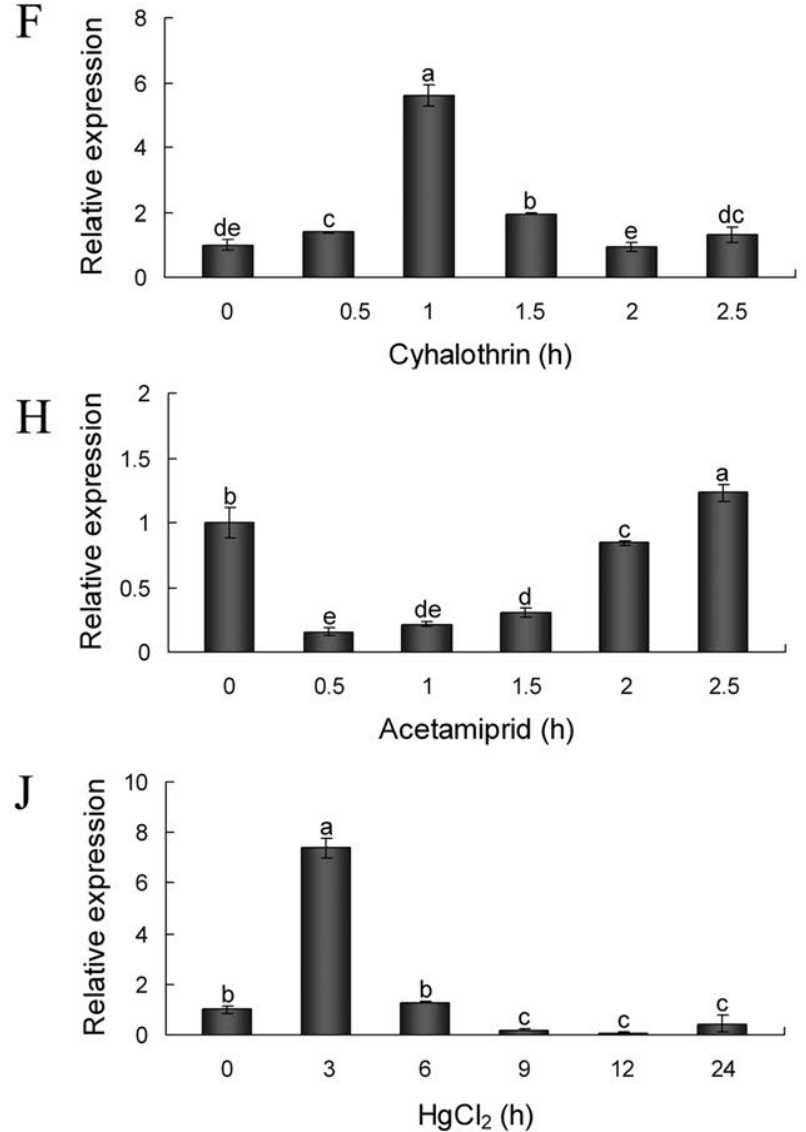

Fig. 4. AccGS expression in response to various abiotic stresses. qPCR was performed to detect the relative expression of $A c c G S$ after treatment with cold (A), heat (B), $\mathrm{H}_{2} \mathrm{O}_{2}(\mathrm{C}), \mathrm{UV}(\mathrm{D})$, paraquat (E), cyhalothrin (F), phoxim (G), acetamiprid (H), acaricide (I), and $\mathrm{HgCl}_{2}(\mathrm{~J})$ treatments. The expression levels were normalized to those in untreated bees $(0 \mathrm{~h})$. The vertical bars represent the mean \pm S.E.M. $(n=3)$. The different letters above the columns indicate significant differences $(P<0.01)$ according to Duncan's multiple range tests, calculated SAS version 9.1 software.

a small amount of $A c c G S$ induction was observed upon $\mathrm{H}_{2} \mathrm{O}_{2}$ treatment. Interestingly, Acc $G S$ expression was significantly induced at $2 \mathrm{~h}$ after exposure to UV light. Pesticides and heavy metals are harmful to the nervous system and can cause neurodegenerative diseases (Franco et al., 2009). To investigate whether AccGS expression is affected by exposure to pesticides/herbicides and mercury, five types of commercial pesticide/herbicide as well as $\mathrm{HgCl}_{2}$ were fed to worker bees (Fig. 4E-J). All treatments increased $A c c G S$ expression, although the induction times varied. The observed elevation of $A c c G S$ 

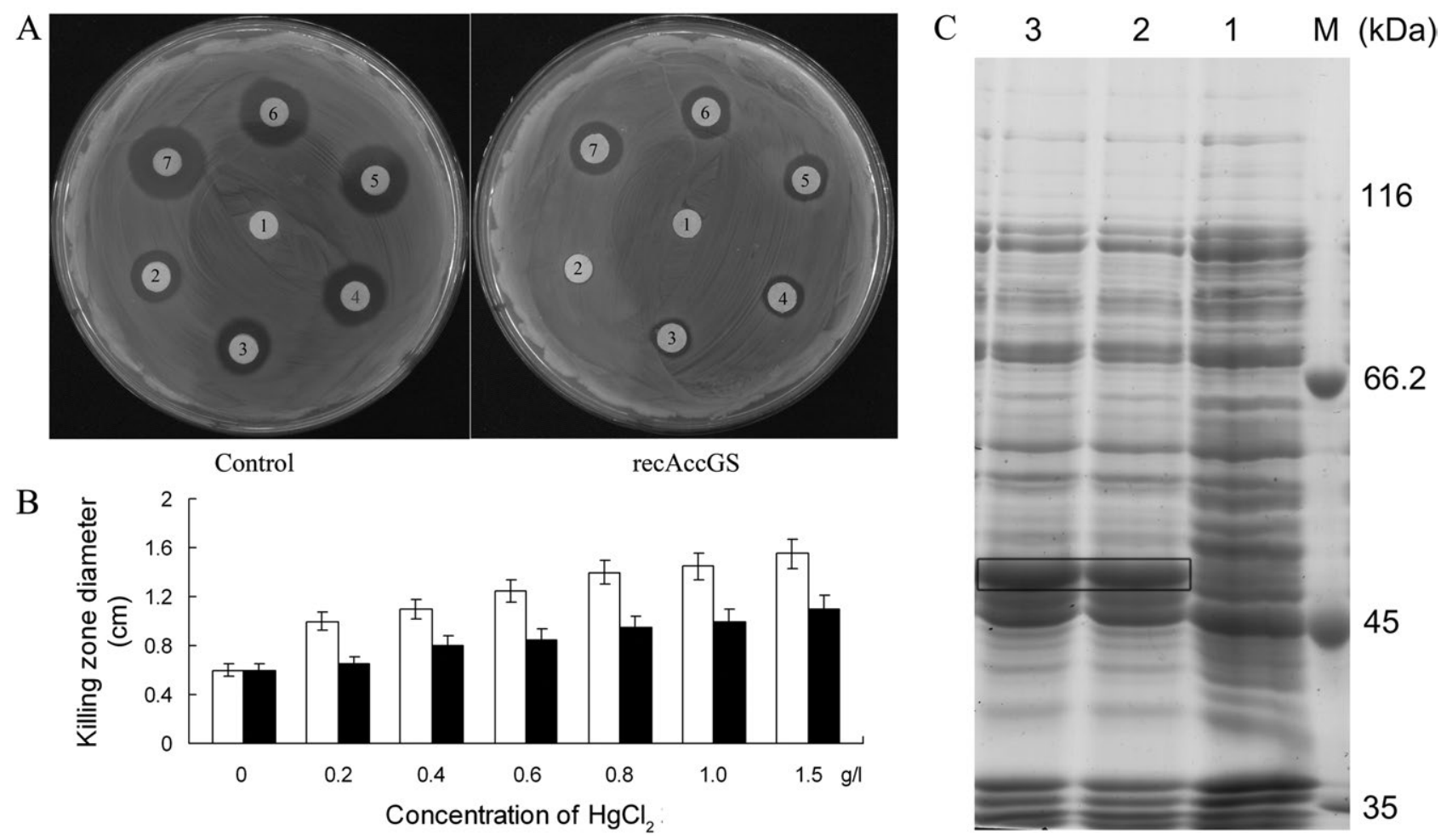

Fig. 5. A disc diffusion assay testing the effect of recAccGS on survival of $\mathrm{HgCl}_{2}$ stress and expression of the recAccGS. (A) Filter discs soaked with different concentrations of $\mathrm{HgCl}_{2}$ were placed on agar plates overnight at $37^{\circ} \mathrm{C} .1: \mathrm{H}_{2} \mathrm{O} ; 2: 0.2 \mathrm{~g} / 1 ; 3: 0.4 \mathrm{~g} / \mathrm{l}$; 4: $0.6 \mathrm{~g} / \mathrm{l} ; 5: 0.8 \mathrm{~g} / \mathrm{l} ; 6: 1 \mathrm{~g} / \mathrm{l}$; and $1.5 \mathrm{~g} / 1 \mathrm{HgCl}_{2}$. The data shown are the means $\pm \mathrm{SE}(\mathrm{n}=3)$ of three independent experiments. (B) Growth inhibition zone diameters. The vertical bars represent the means \pm S.E.M. $(\mathrm{n}=3)$. The different letters above the columns indicate significant differences $(P<0.01)$ according to Duncan's multiple range tests, calculated using SAS version 9.1 software. (C) Sodium dodecyl sulphate-polyacrylamide gel electrophoresis (SDS-PAGE) analysis was used to analyze recombinant $A c c$ GS protein expressed in Escherichia coli BL21. Lane M, Marker in $\mathrm{kDa}$; lanes 1, non-induced overexpression of pET-30a (+)-AccGS in BL21. lanes 2 and 3, induced overexpression of pET-30a (+)-AccGS in BL21. The induced AccGS recombinant protein was boxed.

expression suggested that $A c c G S$ might be a critical factor in abiotic stress responses.

To further confirm the ability of $A c c G S$ to protect against heavy metal stress, E. coli cells in which AccGS overexpression (Fig. 5C) was driven by IPTG induction were exposed to $\mathrm{HgCl}_{2}$. The growth inhibition zones containing no $A c c G S$-overexpressing E. coli around $\mathrm{HgCl}_{2}-$ soaked filters on plates were measured after overnight exposure. The growth inhibition zones of the bacteria expressing recAccGS were smaller than those of the control bacteria (Fig. 5A-B).

\section{DISCUSSION}

GS is essential for the detoxification process of ammonia and plays roles in responses to stress (Peh et al., 2010). Because the function of GS in hymenopteran insects has been poorly documented, we isolated a GS gene from A. c. cerana. Multiple alignments showed that AccGS has high homology to GSs in other species (Fig. 1A). Moreover, AccGS shares 66\% sequence similarity with human GS, which is involved in clearing excitatory glutamate in the brain and ammonia detoxification in the liver (Adeva et al., 2012), suggesting that the role of $A c c G S$ may be similar to the roles of GS in other organisms.
In many species, GS synthesis is regulated during embryonic development. In rats, GS production was detected at 14 days of development (Mearow et al., 1989). In the sea urchin, GS is expressed in the egg and is developmentally regulated in the embryo (Fucci et al., 1995). Similarly, our expression analysis at different developmental stages showed that $A c c G S$ was more highly expressed in the sixth larval instar and white-eyed pupae than in other stages (Fig. 3C). Congenital GS deficiency causes neonatal death and brain malformations in humans and mice (Häberle et al., 2005; He et al., 2010). Indeed, brain tissue, which is responsible for learning, memory, and olfactory signalling, is very sensitive to toxicants (Ament et al., 2008). The honeybees used in the present experiment are social insects with a sophisticated brain and a highly regulated neuronal network. The tissue-specific expression analysis showed that the highest levels of $A c c G S$ transcript were expressed in the brain (Fig. 3A, B). GS plays a central role in the detoxification of ammonia in the brain and in the metabolic regulation of the neurotransmitter glutamate (Suárez et al., 2002). Furthermore, Cro, a brain development-related transcriptional activator-binding motif, was also found in the 5 ' flanking region of $A c c G S$. The strong accumulation of $A c c G S$ in the sixth larval instar and brain suggests that $A c c G S$ plays a role in early development and the brain. 
There is increasing evidence that GS enzymes perform functions in detoxifying xenobiotics and oxidative damage (David et al., 2005; Chen \& Herrup, 2012) and that GSs can be induced by xenobiotics, such as pesticides and hydrocarbons, which can release nitrogen as an ammonium group. In addition, the majority of the enzymes related to xenobiotic detoxification releases an ammonium group and enhance the catabolic functions of various proteins; furthermore, those groups can also activate GS (Tanguy et al., 2005b). In our experiment, AccGS expression was induced by all the pesticides/herbicides tested (Fig. 4E-I). This finding is consistent with previous studies showing that aquatic organisms exposed to pesticides and hydrocarbons exhibit increased GS expression (Boutet et al., 2004; Tanguy et al., 2005a). Furthermore, we report the first evidence of a role for AccGS in the response to environmental stresses such as cold, heat, UV light, $\mathrm{H}_{2} \mathrm{O}_{2}$ and $\mathrm{HgCl}_{2}$ (Fig. 4A-D, J), showing that $A c c G S$ could be induced by the above stressors. The toxicity of pro-oxidants, temperature, heavy metals, and pesticides is thought to occur by causing the excessive production or accumulation of reactive oxygen species (ROS) (Narendra et al., 2007; Brennan et al., 2008), which damages DNA and proteins. Moreover, under conditions of low glutamine, the levels of multiple stress response proteins are reduced, rendering cells hypersensitive to $\mathrm{H}_{2} \mathrm{O}_{2}$ and DNA damage (Chen \& Herrup, 2012). Thus, we suggest that $A c c G S$ is involved in oxidative damage. The significant difference in the size of the growth inhibition zones (Fig. 5A, B) between the AccGS-overexpressing and control bacteria provides direct evidence that AccGS contributes to the cellular resistance to heavy metals. Several HSF elements were also found in the $5^{\prime}$ flanking region of AccGS. Taken together, the results demonstrating the accumulation of $A c c G S$ upon exposure to various stressors argue that $A c c G S$ plays important roles in the protection against abiotic stresses by maintaining glutamine levels. Furthermore, our characterization of $A c c G S$ suggests that the gene plays important roles in early development and abiotic stress responses, providing important information about $G S$ genes in different animal species.

ACKNOWLEDGEMENTS. This work was financially supported by the earmarked fund for Modern Agro-industry Technology Research System (No. CARS-45) and the Special Fund for Agro-scientific Research in the Public Interest (No. 200903006).

\section{REFERENCES}

Adeva M.M., Souto G., Blanco N. \& Donapetry C. 2012: Ammonium metabolism in humans. - Metabolism 61: 1495-1511.

Ament S.A., Corona M., Pollock H.S. \& Robinson G.E. 2008: Insulin signaling is involved in the regulation of worker division of labor in honey bee colonies. - Proc. Natl. Acad. Sci. U. S. A. 105: 4226-4231.

Boutet I., Tanguy A. \& Moraga D. 2004: Response of the Pacific oyster Crassostrea gigas to hydrocarbon contamination under experimental conditions. - Gene 329: 147-157.
Brennan L.J., Keddie B.A., Braig H.R. \& Harris H.L. 2008: The endosymbiont Wolbachia pipientis induces the expression of host antioxidant proteins in an Aedes albopictus cell line. - PLOS ONE 3: e2083.

Chen J. \& Herrup K. 2012: Glutamine acts as a neuroprotectant against DNA damage, beta-amyloid and $\mathrm{H}_{2} \mathrm{O}_{2}$-induced stress. - PLOS ONE 7: e33177.

David E., Tanguy A., Pichavant K. \& Moraga D. 2005: Response of the Pacific oyster Crassostrea gigas to hypoxia exposure under experimental conditions. - FEBS J. 272: $5635-5652$.

Ericsson A., Kotarsky K., Svensson M., Sigvardsson M. \& AgACE W. 2006: Functional characterization of the CCL25 promoter in small intestinal epithelial cells suggests a regulatory role for caudal-related homeobox $(\mathrm{Cdx})$ transcription factors. - J. Immunol. 176: 3642-3651.

Fernandes M., XIAo H. \& Lis J.T. 1994: Fine structure analyses of the Drosophila and Saccharomyces heat shock factor-heat shock element interactions. - Nucl. Acids Res. 22: 167-173.

Franco R., Sánchez-Olea R., Reyes-Reyes E.M. \& PanayioTIDIS M.I. 2009: Environmental toxicity, oxidative stress and apoptosis: ménage à trois. - Mutat. Res. 674: 3-22.

Fu Y.H. \& MARZLUF G.A. 1990: nit-2, the major nitrogen regulatory gene of Neurospora crassa, encodes a protein with a putative zinc finger DNA-binding domain. - Mol. Cell Biol. 10: $1056-1065$.

Fucci L., Piscopo A., Aniello F., Branno M., Gregorio A.D., CAlogero R. \& Geraci G. 1995: Cloning and characterization of a developmentally regulated sea urchin cDNA encoding glutamine synthetase. - Gene 152: 205-208.

Geret F., Burgeot T., Haure J., Gagnaire B., Renault T., ComMUNAL P.Y. \& SAMAIN J.F. 2013: Effects of low-dose exposure to pesticide mixture on physiological responses of the Pacific Oyster, Crassostrea gigas. — Environ. Toxicol. 28: 689-699.

Häberle J., Görg B., Rutsch F., Schmidt E., Toutain A., Benoist J.F., Gelot A., Suc A.L., Höhne W., Schliess F., HäUsSinger D. \& Koch H.G. 2005: Congenital glutamine deficiency with glutamine synthetase mutations. - N. Engl. J. Med. 353: 1926-1933.

He Y., Hakvoort T.B., Vermeulen J.L., Labruyère W.T., De WaArt D.R., Van Der Hel W.S., RuiJter J.M., Uylings H.B. \& LAMERS W.H. 2010: Glutamine synthetase deficiency in murine astrocytes results in neonatal death. - Glia 58: 741-754.

Jefrerey P.L., Capes-Davis A., Dunn J.M., Tolhurst O., Seeto G., Hannan A.J. \& Lin S.L. 2000: CROC-4: a novel brain specific transcriptional activator of c-fos expressed from proliferation through to maturation of multiple neuronal cell types. - Mol. Cell Neurosci. 16: 185-196.

Kinoshita S., Isu S., KaneKo G., Yamada H., Hara T., Itoh Y. $\&$ WatABE S. 2009: The occurrence of eukaryotic type III glutamine synthetase in the marine diatom Chaetoceros compressum. - Mar. Genomics 2: 103-111.

Kosenko E.A., Venediktova N.I., Kudryavtsev A.A., AtaullaKhanov F.I., Kaminsky Y.G., Felipo V. \& Montoliu C. 2008: Encapsulation of glutamine synthetase in mouse erythrocytes: a new procedure for ammonia detoxification. - Biochem. Cell Biol. 86: 469-476.

Kumada Y., Benson D.R., Hillemann D., Hosted T.J., Rochefort D.A., Thompson C.J., Wohlleben W. \& Tateno Y. 1993: Evolution of the glutamine synthetase gene, one of the oldest existing and functioning genes. - Proc. Natl. Acad. Sci. U.S.A. 90: 3009-3013.

Lai X.F., Gao H., Kong J., Wang Q.Y., Wang W.J. \& Meng X.H. 2011: Cloning and characterization of the glutamine 
synthetase gene from Chinese shrimp Fenneropenaeus chinensis. - Aquacult. Int. 19: 873-889.

Liu D. \& Chen Z. 2011: The regulatory effects of glutamine on illness and health. - Protein Pept. Lett. 18: 658-662.

Mearow K.M., Mill J.F. \& Vitkovic L. 1989: The ontogeny and localization of glutamine synthetase gene expression in rat brain. - Brain Res. Mol. Brain Res. 6: 223-232.

Meng F., Kang M., Liu L., Luo L., Xu B. \& Guo X. 2011: Characterization of the TAKl gene in Apis cerana cerana (Acc$T A K 1)$ and its involvement in the regulation of tissue-specific development. - BMB Rep. 44: 187-192.

Mullin C.A., Frazier M., Frazier J.L., Ashcraft S., Simonds R., van Engelsdorp D. \& Pettis J.S. 2010: High levels of miticides and agrochemicals in North American apiaries: implications for honey bee health. — PLOS ONE 5: e9754.

Narendra M., Bhatracharyulu N.C., Padmavathi P. \& Varadacharyulu N.C. 2007: Prallethrin induced biochemical changes in erythrocyte membrane and red cell osmotic haemolysis in human volunteers. - Chemosphere 67: 1065-1071

Pen W.Y., Chew S.F., Ching B.Y., Loong A.M. \& IP Y.K. 2010 Roles of intestinal glutamate dehydrogenase and glutamine synthetase in environmental ammonia detoxification in the euryhaline four-eyed sleeper, Bostrychus sinensis. - Aquat. Toxicol. 98: 91-98.

Qiu C.H., Hong Y., Cao Y., Wang F., Fu H.Q., Shi Y., Wei M., LiU S. \& LiN J.J. 2012: Molecular cloning and characteriza- tion of glutamine synthetase, a tegumental protein from Schistosoma japonicum. - Parasitol. Res. 111: 2367-2376.

SANDERS M.M. \& Kon C. 1992: Glutamine and glutamate metabolism in normal and heat shock conditions in Drosophila Kc cells: conditions supporting glutamine synthesis maximise heat shock polypeptide expression. - J. Cell Physiol. 150: 620-631.

SuÁrez I., Bodega G. \& Fernández B. 2002: Glutamine synthetase in brain: effect of ammonia. - Neurochem. Int. 41: $123-142$.

Tanguy A., Boutet I., Laroche J. \& Moraga D. 2005a: Molecular identification and expression study of differentially regulated genes in the Pacific oyster Crassostrea gigas in response to pesticides exposure. - FEBS J. 272: 390-403.

Tanguy A., Boutet I. \& Moraga D. 2005b: Molecular characterization of the glutamine synthetase gene in the Pacific oyster Crassostrea gigas: expression study in response to xenobiotic exposure and developmental stage. - Biochim. Biophys. Acta 1681: 116-125.

Weitzel L.R. \& Wischmeyer P.E. 2010: Glutamine in critical illness: the time has come, the time is now. - Crit. Care Clin. 26: 515-525.

Xu P., Shi M. \& Chen X.X. 2009: Antimicrobial peptide evolution in the Asiatic honey bee Apis cerana. - PLoS ONE 4: e4239.

Received May 11, 2013; revised and accepted August 9, 2013 